\title{
An evaluation of a pilot study of a web-based educational initiative for educating and training undergraduate dental students in infection prevention
}

IN BRIEF

- Provides a model for delivery of infection prevention and control education for dental undergraduates.

- Outlines assessment of the first cohort of undergraduates to use the pilot model of infection prevention education.

- Provides learning outcomes for infection prevention and education for dental undergraduates.

\author{
D. E. A. Lockhart ${ }^{1}$ and A. J. Smith ${ }^{2}$
}

\begin{abstract}
Objective Evaluate the views of undergraduate dental students on a pilot web-based e-learning programme to establish its merit in providing education in infection prevention and control. The initiative was commissioned by NHS Education for Scotland as an educational resource for healthcare workers. Design, sample and setting This was a retrospective analysis of questionnaire data from the first cohort of dental students completing the programme in a UK dental school. Methods Dental students studied the course during the first three years of the curriculum. Data were collated via an anonymous semi-structured questionnaire distributed at a post-course feedback session. Results Sixty percent (51/85) of students returned questionnaires with $90 \%$ stating their knowledge of infection control had improved. Specific areas included: undertaking infection control audits (88\%), managing sharps injuries (73\%), use of personal protective equipment (55\%) and hand hygiene (55\%). On the other hand, 72\% perceived the programme as too generic with just over half advocating a resource more relevant to dentistry. Conclusion The programme has the potential to be a useful teaching aid but requires modification. A customised version for dental students has subsequently been commissioned.
\end{abstract}

\section{INTRODUCTION}

Dental undergraduates require knowledge of infection prevention and control to fulfil their professional obligations of providing safe and efficient patient care as prescribed in the General Dental Council's (GDC) The first five years. ${ }^{1}$ Previous work ${ }^{2,3}$ stressed the importance of educational interventions in promoting high standards in issues surrounding infection control. Yet devising an innovative approach delivering these messages and engaging students presents a challenge. ${ }^{3}$ The advent of e-learning has seen a shift in teaching styles from traditional 'lecturing and telling' to 'facilitating and guiding'.

A national survey ${ }^{5}$ demonstrated several shortcomings in the application and

1*Specialist Registrar/Honorary Clinical Teacher Microbiology, ${ }^{2}$ Senior Clinical Lecturer/Honorary Consultant Microbiologist, Infection \& Immunity, University of Glasgow Dental Hospital \& School, Faculty of Medicine, 378 Sauchiehall Street, Glasgow G2 3JZ

Correspondence to: Deborah E. A. Lockhart Email:d.lockhart@dental.gla.ac.uk

\section{Refereed Paper}

Accepted 2 July 2009

DOI: $10.1038 /$ sj.bdj.2009.765

${ }^{\oplus}$ British Dental Journal 2009; 207: 223-226 delivery of infection prevention and control in general dental practice. These results prompted an internal critical review of infection control and instrument decontamination training and revealed insufficient breadth and depth in this important area, for instance, an absence of practical skills in cleaning and sterilising dental instruments. The undergraduate BDS curriculum at the University of Glasgow was completely revised in 2004 allowing scope for the introduction of a new initiative. In summary, infection prevention and control is now subdivided into the basic principles of standard infection control procedures (SICPs) delivered in years 1-3 followed by an applied programme focused on instrument decontamination in years 3-5.

Promoting the Prevention and Control of Infection through Cleanliness Champions (CC) is a web-based e-learning initiative commissioned by NHS Education for Scotland (NES) in response to the Scottish Government's Action Plan on reducing healthcare associated infections (HAI). ${ }^{6}$ The programme is designed to be selfdirected with some mentorship support and apply to all healthcare workers. Based on
SICPs (Table 1) it combines narrative with online questions, clinical activities and reflective analyses.

The University of Glasgow, in collaboration with NES, identified the potential benefits of putting a national policy into practice and is the first dental school to pilot CC in the undergraduate curriculum. This paper describes the logistics of introducing CC and presents the views of the first cohort of dental students completing the programme to establish its merit in providing education in infection prevention and control. It should be emphasised that the CC programme does not provide education on the practical operation and management of a local decontamination unit.

The introduction of the instrument decontamination training programme is discussed in a subsequent article.

\section{METHOD}

\section{Introduction into the undergraduate curriculum}

CC formed a component of Clinical Medical Sciences in the integrated BDS curriculum. All users were issued with a unique username 


\begin{tabular}{l|l|}
\hline \multicolumn{2}{|l}{ Table 1 Standard infection control } \\
procedures apply in all healthcare settings \\
\hline & Standard infection control procedures \\
\hline 1 & Hand hygiene \\
\hline 2 & Personal protective equipment \\
\hline 3 & Prevention of occupational exposure \\
\hline 4 & Management of blood and body fluid spillage \\
\hline 5 & Cleanliness of care equipment \\
\hline 6 & Cleanliness of the environment \\
\hline 7 & Safe handling of linen \\
\hline 8 & Safe disposal of waste \\
\hline 9 & Patient placement \\
\hline
\end{tabular}

and password to access the online material located on an external secure server (Fig. 1). Weblinks were provided via a dedicated CC resource area on the dental school intranet.

Students were inducted into CC by an introductory session at the start of year 1 and studied the programme in a linear fashion for the first three years of the curriculum (Fig. 2). While primarily self-directed, lectures, practical classes and an online discussion forum complemented the webbased material. Evidence of attainment of the learning outcomes (Table 2) was demonstrated by submission of a 'Folder of Evidence' consisting of a print-out of the students responses to the prescribed activities in each unit. These were scrutinised by a group of mentors following the University of Glasgow's code of assessment. A pass in each unit was required to allow progression to the next academic year.

\section{Evaluation}

All third-year students attending a postcourse feedback session were invited to submit an anonymous semi-structured questionnaire relating to their perceived application of infection control in clinical practice and the programme itself. The questionnaire was divided into two parts with a range of closed and open questions. Only one answer was permitted in the closed questions, however, open questions allowed elaboration of these areas in an unrestricted format.

\section{RESULTS}

Questionnaires were returned by $51 / 85$ students giving a response rate of $60 \%$. On average 42 students contributed to the open

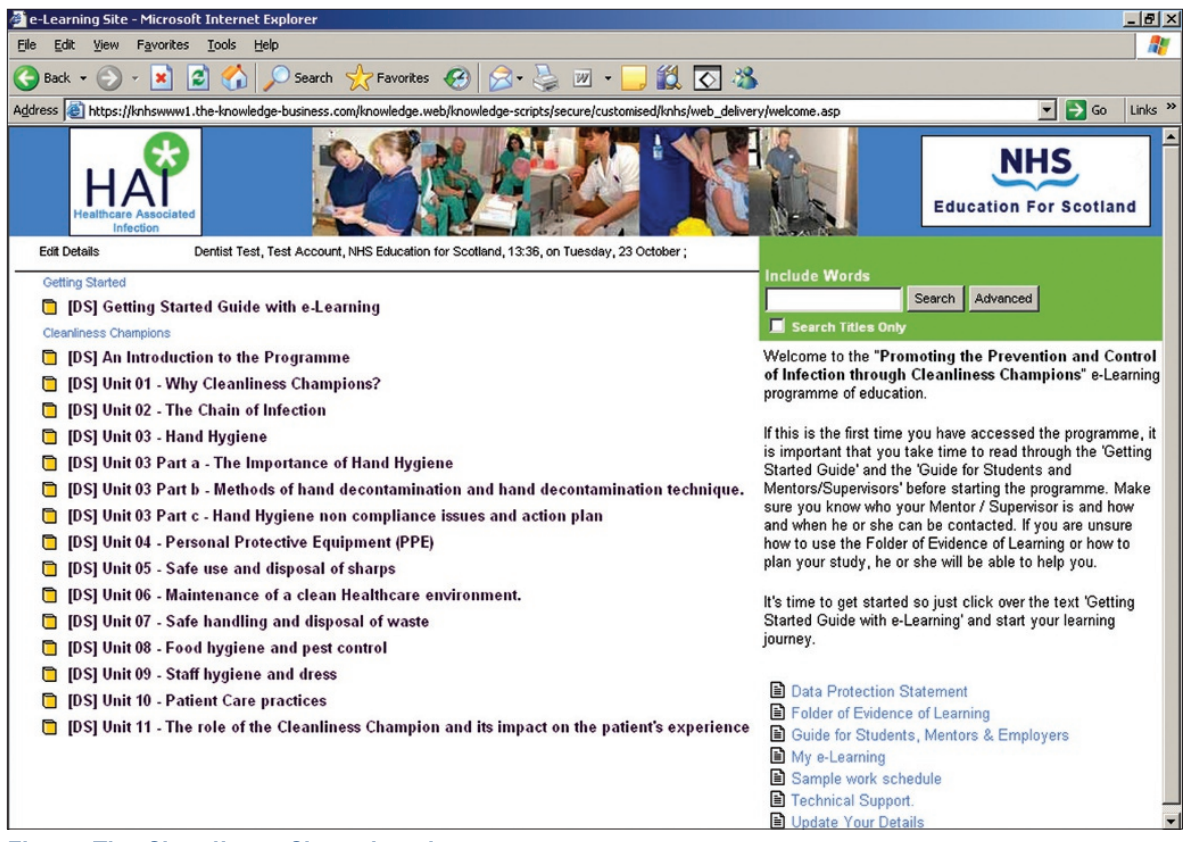

Fig. 1 The Cleanliness Champions homepage

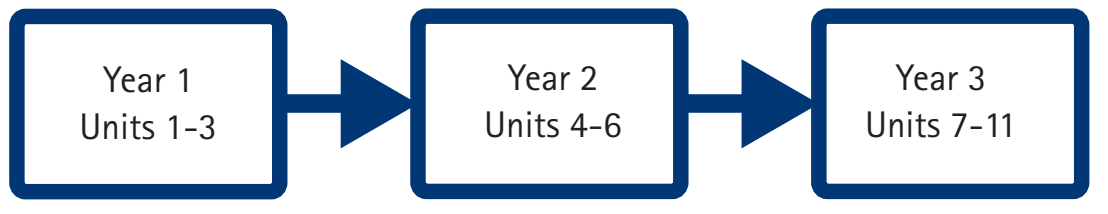

Fig. 2 Placement of Cleanliness Champions in the undergraduate curriculum

Table 2 Overall learning outcomes (individual units are supported by specific learning outcomes and competencies)

\begin{tabular}{|c|c|}
\hline & Learning outcomes \\
\hline 1 & $\begin{array}{l}\text { Appreciate the role of the Cleanliness Champion in promoting a safety culture within the } \\
\text { healthcare setting. }\end{array}$ \\
\hline 2 & Describe the core principles of infection prevention and control. \\
\hline 3 & $\begin{array}{l}\text { Explain the reasons for adhering to policies and procedures relating to infection prevention } \\
\text { and control. }\end{array}$ \\
\hline 4 & $\begin{array}{l}\text { Describe the Chain of Infection and demonstrate an understanding of the interventions necessary } \\
\text { to prevent and control infection. }\end{array}$ \\
\hline 5 & Demonstrate positive role modelling by promoting safe practice and a safe environment for patients. \\
\hline 6 & Conduct audits using recognised tools. \\
\hline 7 & Develop the core competencies described in the programme. \\
\hline
\end{tabular}

questions and selected comments are shown in italics. Ninety percent of respondents stated their knowledge of infection control had improved (Table 3). Specific areas of clinical practice enhanced (Fig. 3) included the ability to perform audits (88\%) and manage sharps injuries (73\%).

On the other hand, $72 \%$ felt CC was unsuitable for dental students due to the inclusion of material perceived irrelevant to dentistry, for example, aseptic techniques in handling urinary catheters. In relation to the open questions, a range of comments were made with the majority (76\%) suggesting CC should be adapted for dentistry and the units streamlined. Most (70\%) agreed the programme should be commenced in year 1 but opinions were divided over the length of the course with 55\% thinking it could be completed entirely in first year.

\section{DISCUSSION}

\section{Infection control education}

Before the introduction of an integrated BDS curriculum in 2004, infection control 


\begin{tabular}{|c|c|c|c|}
\hline Cleanliness Champions... & $\begin{array}{l}\text { Yes } \\
(\%)\end{array}$ & $\begin{array}{l}\text { No } \\
(\%)\end{array}$ & $\begin{array}{l}\text { N/A } \\
(\%)\end{array}$ \\
\hline Improved your knowledge of infection control? & 90 & 8 & 2 \\
\hline Changed your attitude to infection control? & 61 & 39 & 0 \\
\hline Is suitable for dental undergraduates? & 24 & 72 & 4 \\
\hline Is started at the right time in the BDS curriculum? & 71 & 29 & 0 \\
\hline Is the right length (spread over three years)? & 45 & 51 & 4 \\
\hline Should be longer (eg spread over five years)? & 4 & 94 & 2 \\
\hline Should be shorter (eg spread over one year)? & 55 & 43 & 2 \\
\hline Should be delayed until final year? & 0 & 100 & 0 \\
\hline Online activities were useful? & 39 & 59 & 2 \\
\hline Workplace activities were useful? & 39 & 59 & 2 \\
\hline
\end{tabular}

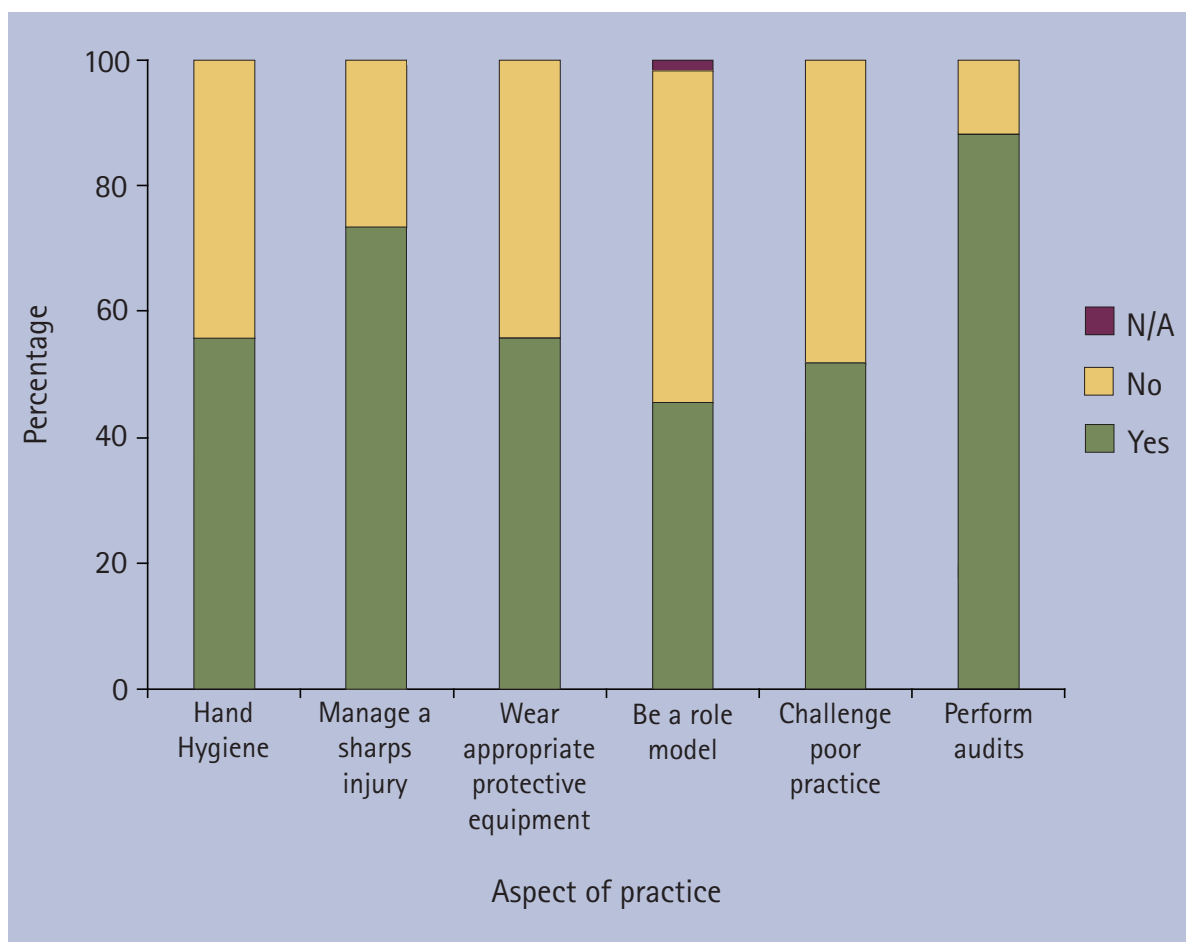

Fig. 3 Ability to alter clinical practice

education in Glasgow was predominantly didactic comprising lectures and issuing the dental hospital infection control policy. As guidelines and policies continuously evolve, future dentists require transferable skills to facilitate professional development in this area. Thoroughly revising the infection control teaching demonstrated commitment to pursue effective educational changes. Indeed evidence of adequate disinfection and decontamination procedures now form part of the GDC revalidation scheme. ${ }^{7}$

'Infection control is everybody's business' ${ }^{8}$ and the principles of good practice must be ingrained early in undergraduate training to avert issues of poor compliance and negative attitudes. ${ }^{2,3,9}$ Milward \&t Cooper ${ }^{10}$ reported positive results following formal infection control competency assessments for dental undergraduates. This together with our findings demonstrates this subject must have specific learning outcomes and competencies in the curriculum. To stress importance in an assessment driven culture, the material was also eligible for inclusion in written professional examinations and objective structured clinical examinations (OSCE).

\section{Logistics of introducing} a new programme

Although educationalists ${ }^{11}$ speculate there will be a greater emphasis on 'blended learning' combining self-directed and face-to-face teaching methods, concerns have been raised how these educational processes are quality assured. As dental schools struggle with a shortage of academic staff, the onus of responsibility for infection control education is shared between clinicians, microbiologists and infection control nurses.

Acknowledging this, an e-learning approach delivered innovation in infection control education by offering the ability to choose the time, place and pace of study. Students were allocated protected time and had access to computers in the dental school and main university library. The use of e-learning in dentistry has been favourable ${ }^{12,13}$ although there is a consensus it should complement rather than replace traditional teaching methods. This may manifest in more feedback sessions where guidance and model answers can be discussed.

One of the obstacles in administering CC was recruiting and retaining suitable mentors to assess the 'Folder of Evidence' to provide students with support and feedback. Ideally, mentors should be recruited from the students workplace, however, we encountered a disappointing lack of support from clinicians within the dental school to act as mentors. As a result volunteers were recruited from infection control nurses or consultants/specialist registrars in microbiology based outside the dental hospital. Although one-to-one mentoring was not logistical, the students had access to an online discussion forum moderated by a 'virtual mentor' (DL). Face-to-face contact was provided via 'drop-in' sessions but these were poorly attended and later abandoned.

Mentorship is an ongoing commitment and each received approximately six folders nine times per year corresponding to the submission deadlines for each year cohort. Each batch took approximately three hours to assess and added to the administrative workload for staff. The quality of the work varied tremendously but as an incentive prizes were awarded for selected units.

\section{What did the students think?}

Previous questionnaire evaluation demonstrated CC is useful in a variety of 
healthcare settings. ${ }^{14}$ Our own observations confirm this with over $60 \%$ stating their attitude to infection control changed and it 'challenged me to think about what I am doing' and made them 'more vigilant about washing hands'.

Workplace activities provided a valuable opportunity to audit clinical practice, such as hand hygiene, and interact with members of the dental team. Although such educational interventions are sometimes perceived as 'patronising', others ${ }^{9}$ have shown only $8.5 \%$ of medical students demonstrated adequate hand hygiene following patient contact and 58\% knew the correct indications for alcohol gel. ${ }^{15}$ Moreover, students were required to develop investigative skills in sourcing relevant policies and information. This aspect is likely to have been neglected previously and may account for a proportion of practising dentists being unfamiliar with standard policies. ${ }^{16}$

Devising a programme flexible enough to accommodate all healthcare professionals is a challenge as reflected by the less favourable feedback.

Students stated that 'many of the important points were drowned out with useless info' and that it was 'designed and worded with nurses in mind'. Perhaps at this stage of their career they may be unaware of the role of dentistry in the wider healthcare context.

Just under half (45\%) requested 'a programme designed specifically for dental students'. These viewpoints were comparable with other pilot studies featuring undergraduate medical and nursing students. ${ }^{17,18}$ Effectively NES created a standard e-learning initiative that can be customised for specific groups, for example dentists and medics, while retaining basic principles and its original authenticity and presentation.

Finally, data collection via post-evaluation questionnaires suffers from a lack of objectivity and may result in memory bias. ${ }^{2}$ Additionally viewpoints may be skewed by the impression that dedicated teaching in infection prevention and control is the norm but in a UK medical school almost half the students expressed concerns that there was insufficient emphasis on this area. ${ }^{15}$

Anomalies were demonstrated by the trend that 59\% did not find the workplace activities useful yet on the other hand 88\% stated their ability to audit clinical practice had been improved. Similarly, when asked to comment on areas of practice most influenced by CC, hand hygiene was most frequently reported compared to 55\% in the closed questions. Thus self-reported compliances may not reflect actual behaviour.

\section{CONCLUSIONS}

Despite some perceived resistance, as is often evident when introducing any new initiative, we conclude CC has made a positive impact and 'has the potential to be very useful'. In taking matters forward, modifications to the existing programme are evidently required. But there must also be dedicated resources to supply and support appropriately qualified mentors to assess the students' progress and work. Ideally, supervising clinicians from the dental school are best placed to mentor undergraduates in this core professional topic and also benefit themselves from continuing professional development. The data gathered from this evaluation provides evidence for developing a version tailored specifically for dental healthcare workers and a revised version has subsequently been introduced.

This initiative would not have succeeded without the 'volunteering' of the mentors: Jeremy Bagg, John Boyes, Margaret Brown, Ben Cooke, Kirsty Ferguson, Kate Hamilton, Saranaz Jamdar, Nitish Khanna, David Lappin, Mairi MacLeod, Jackie McIntyre, Sandra McNamee, Libby Patterson, Gordon Ramage, Jackie Stewart, Bishan Thakker and Pauline Wright. We also wish to thank Laetitia Brocklebank for initial discussions, NHS Education for Scotland, The Knowledge Business, the University of Glasgow and dental students who participated in the pilot study.
1. The first five years, 3rd ed (interim 2008). A framework for undergraduate dental education. London: General Dental Council, 2008.

2. Martins T C, Ramos-Jorge M L, Magela-Machado D, Paiva S M et al. Management of occupational bloodborne exposure in a dental teaching environment. J Dent Educ 2007; 71: 1348-1355.

3. Borges-Yáñez S A, Flores M et al. Infection control attitudes and perceptions among dental students in Latin America: implications for dental education. Int Dent J 2008; 58: 187-193.

4. Reynolds P A, Mason R, Eaton K A. Remember the days in the old school yard: from lectures to online learning. Br Dent J 2008; 204: 447-451.

5. NHS Scotland: Sterile Services Provision Review Group. Survey of decontamination in general dental practice. Edinburgh: The Stationery Office, 2004. http://www.scotland.gov.uk/ Publications/2004/11/20093/45220.

6. Scottish Executive Health Department. Preventing infections acquired while receiving healthcare. The Scottish Executive's action plan to reduce the risk to patients, staff and visitors 2002-2005. Edinburgh: The Stationery Office, 2002. http://www.scotland.gov.uk/library5/health/ preventinfect.pdf.

7. Revalidation Working Group Report and Consultation. London: General Dental Council, 2008.

8. Healthcare Associated Infection Task Force. The NHS Scotland code of practice for the local management of hygiene and healthcare associated infection. Edinburgh: The Stationery Office, 2004. http://www.scotland.gov.uk/ Publications/2004/05/19315/36631.

9. Feather A, Stone S P, Wessier A, Boursicot K A, Pratt C. 'Now please wash your hands': the handwashing behaviour of final MBBS candidates. J Hosp Infect 2000; 45: 62-64.

10. Milward M R, Cooper P R. Competency assessment for infection control in the undergraduate dental curriculum. Eur J Dent Educ 2007: 11: 148-154.

11. Eaton K A, Reynolds P A, Grayden S K, Wilson N H. $A$ vision of dental education in the third millennium. Br Dent J 2008; 205: 261-271.

12. Gupta B, White D A, Walmsley A D. The attitudes of undergraduate students and staff to the use of electronic learning. Br Dent J 2004; 196: 487-492.

13. Reynolds P A, Rice S, Uddin M. Online learning in dentistry: the changes in undergraduate perceptions and attitudes over a four year period. Br Dent J 2006; 203: 419-423.

14. West B J M, Macduff C, MacBain M, Gass J. An evaluation of the implementation and development of the NHS Education for Scotland cleanliness champions programme. Edinburgh: NES, 2006. http: //www.nes.scot.nhs.uk/documents/publications/ classa/CCP_Evaluation_Full_report_(final).pdf.

15. Mann C M, Wood A. How much do medical students know about infection control? J Hosp Infect 2006; 64: 366-370.

16. Myers $R$, Larson $E_{1}$ Cheng $B$, Schwartz A, Da Silva $K$, Kunzel $C$. Hand hygiene among general practice dentists. A survey of knowledge, attitudes and practices. J Am Dent Assoc 2008; 139: 948-957.

17. Chalmers C, Straub M. Infection control education for undergraduates. Nurs Stand 2006; 20: 35-41.

18. Phillips G, Kerr J. Champion students! Experience with a standardized infection control training package in medical students. J Hosp Infect 2006; 62: 518-519. 